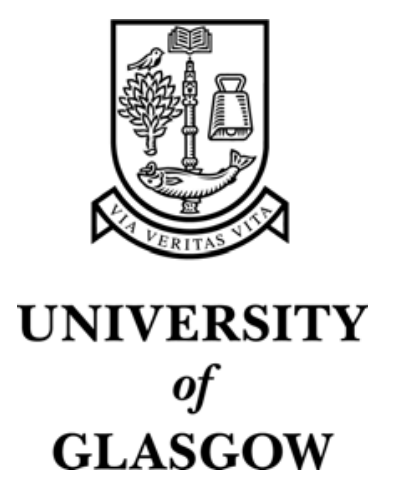

Crossan, A. and Murray-Smith, R. and Brewster, S.A. and Kelly, J. and Musizza, B. (2005) Gait phase effects in mobile interaction. In, Kellogg, W.A. and Zhai, S. and Gale, C. and van der Veer, G.C., Eds. CHI '05 Conference on Human Factors in Computing Systems, 2-7 April 2005, pages pp. 1312-1315, Portland, Oregon.

http://eprints.gla.ac.uk/3217/ 


\section{Gait Phase Effects in Mobile Interaction}

\author{
Andrew Crossan, \\ Roderick \\ Murray-Smith ${ }^{1,2}$ \\ ${ }^{1}$ Hamilton Institute, \\ NUI Maynooth, Ireland. \\ andrew.crossan@nuim.ie \\ rod@dcs.gla.ac.uk
}

\author{
Stephen Brewster, \\ James Kelly \\ ${ }^{2}$ Dept. of Computing \\ Science, \\ University of Glasgow, \\ Scotland \\ stephen@dcs.gla.ac.uk
}

\author{
Bojan Musizza \\ Dept. of Systems \& \\ Control, \\ Institut Jozef Stefan \\ Jamova 39, 1000 Ljubljana \\ Slovenia \\ bojan.musizza@ijs.si
}

\begin{abstract}
One problem evaluating mobile and wearable devices is that they are used in mobile settings, making it hard to collect usability data. We present a study of tap-based selection of on-screen targets whilst walking and sitting, using a PocketPC instrumented with an accelerometer to collect information about user activity at the time of each tap. From these data the user's gait can be derived, and this is then used to investigate preferred tapping behaviour relative to gait phase, and associated tap accuracy. Results showed that users were more accurate sitting than walking. When walking there were phase regions with significantly increased tap likelihood, and these regions had significantly lower error rates, and lower error variability. This work represents an example of accelerometer-instrumented mobile usability analysis, and the results give a quantitative understanding of the detailed interactions taking place when on the move, allowing us to develop better mobile interfaces.
\end{abstract}

\section{Author Keywords}

Accelerometer, targeting, pen-based interfaces, mobile devices, rhythmic interaction, gait effects, instrumented usability.

\section{ACM Classification Keywords}

Usability testing and evaluation, handheld devices and mobile computing, empirical methods quantitative, user studies

\section{INTRODUCTION}

One of the most important areas of research in computing at present is into mobile and wearable devices, such as phones, PDAs and fully wearable computers. Much work is going on into how to design interactions for these devices, given the limited displays and input techniques. Research in this area is difficult as users cannot be sat in a usability lab; to assess an interface design successfully the user must be moving and this makes it difficult to take standard measures of usability. Researchers have proposed a range of additional techniques

Copyright is held by the author/owner(s).

CHI 2005, April 2-7, 2005, Portland, Oregon, USA.

ACM 1-59593-002-7/05/0004. such as distance walked and percentage preferred walking speed to assess usability [3,5], but these are qualitative. A quantitative understanding of the detailed interactions taking place is needed so that we can fully understand how users interact with the devices, and so further improve them.

One common form of input is via a stylus, tapping buttons or other widgets on a touch screen. This is very effective but can be tricky when walking; on-screen targets can be hard to hit when the device is moving as the user walks. Brewster [1] showed a more than $30 \%$ reduction in performance tapping buttons on the display of a PDA when walking compared to sitting. If we understood the interaction better we may be able to design something more robust to movements caused by walking.

Being able to measure more information from a device being carried would allow a more detailed, quantitative assessment of usability. One way to do this would be to add an accelerometer to find out how the device moves when a user is walking. Such an instrumented device would allow us to answer questions that are currently difficult. For example, in which phases of the gait cycle do users tap targets, and does phase affect the mean accuracy and the variability of those taps? It may be that users find it easier to tap during parts of the cycle where the device is more stable. Interface designers might, as discussed in [2], then take advantage of rhythm in interaction such that ease of natural rhythmic use is more important that raw speed of response.

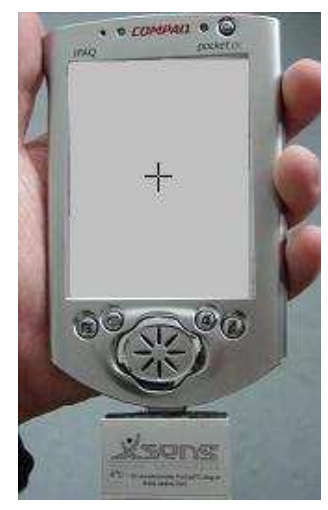

Figure 1. PDA with the Xsens P3C accelerometer attached to the serial port. 
In this paper we show how sensors, like accelerometers, can be used in ways other than for explicit interaction. In this case we use the acceleration data to infer the user's gait, and we investigate whether the rhythm of walking affects the tap timing and error rate of a user selecting targets on screen, while walking and sitting.

\section{EXPERIMENT}

\section{Equipment}

This system was developed using an HP 5550 PDA with the Xsens P3C 3 degree of freedom linear accelerometer attached to the serial port, as shown in Figure 1. Its effect on the balance of the device is negligible (its weight is $10.35 \mathrm{~g}$ ). The accelerometer was used to detect movement of the device, sampling at a rate of approximately $90 \mathrm{~Hz}$.

\section{Task}

The interface used for the study is displayed in Figure 1. Participants were asked to tap on a series of cross-hair targets (drawn 30 pixels high and wide) that were displayed on the screen. There were 15 possible target positions spaced equally around a 3 wide by 5 high grid of positions on the screen. Every second target presented to the participants was the target in the centre of the screen. Each of the other 14 targets was displayed to the user in a random order four times each. The accuracy and speed of tapping were both emphasised as equally important. The position of the tap was recorded as the initial stylus down position on the screen. Once one target had been selected, the next target was displayed a random time interval from 0.5 to 1.5 seconds after the previous selection. This was to prevent rhythm effects affecting the tapping phase information in the mobile condition. There were no restrictions on the accuracy that was required by the user. A tap anywhere on the screen regardless of the position of the target counted as a selection.

There were two experimental conditions: tapping while sitting and tapping while walking and 20 users performed both conditions in a counterbalanced order, with 18 participants being right handed and 2 participants being left handed. All participants tapped with their dominant hand while holding the device in their non-dominant hand. For the walking condition, the participants navigated a quiet triangle of paths on the university campus (of total length approximately 200 metres).

Calibration of the screen becomes an issue when looking at accuracy of tapping in a pen based interface, as an error in the calibration can lead to a consistent and unwanted bias in the results. The screen was calibrated once at the start of the experiment, and the same device was used throughout the experiment. Three participants tested the screen calibration. The device was placed on the desk and users performed a similar task to the tapping study for four separate sessions. In this case accuracy was heavily emphasised as the most important aspect of the study. This was borne out by the much closer concentration of points than in the final results, with mean standard deviation of the error for each participant for all targets being less than a pixel. After each session, the device was rotated by 90 degrees to negate any systematic tapping bias. Mean values were recorded for each screen target position and were subtracted from the final results. This method provides a closer match between the position the user actually tapped in and the recorded tap position.

Comparisons were made between time to tap and accuracy of each of the groups. Time to tap was taken from the time that the target was displayed on the screen to the time of the stylus down event. The hypotheses are that users will be more accurate and faster in the seated condition. The effect of screen position of the target on accuracy of the tap was also examined. In addition, the interactions of participants' tapping and step patterns were examined.

\section{INTERPRETING THE SIGNAL}

As a mobile user walks while holding a mobile device, his or her arm will oscillate as a result of the walking. If we examine only the vertical axis of this oscillation, there will be one oscillation per step. Figure 2 shows a time series for the vertical acceleration axis. A Fast Fourier Transform is used to determine the frequency at which the peak amplitude occurs, between 1 and $3 \mathrm{~Hz}$ in the spectrum. For the controlled conditions in this study, this corresponds to the walking step rate. In practice, this is the frequency of maximum power in the spectrum as the users are trying to hold the device relatively still with respect to their body as they walk. The vertical axis acceleration signal is then zero phase shift filtered using a narrow bandpass Butterworth filter centred around this frequency. Figure 2 demonstrates the filtered signal. As the user walks with the device held steady in one hand, an approximately regular oscillation is formed in the vertical axis. One oscillation corresponds to one step. A Hilbert transform is then used to provide an estimate of the phase information for the filtered signal [4]. This phase plot signal is again shown as the saw-tooth waveform in Figure 2 and can be seen to reset at the lowest point in the signal. This corresponds to the lowest point of the hand in the oscillation.

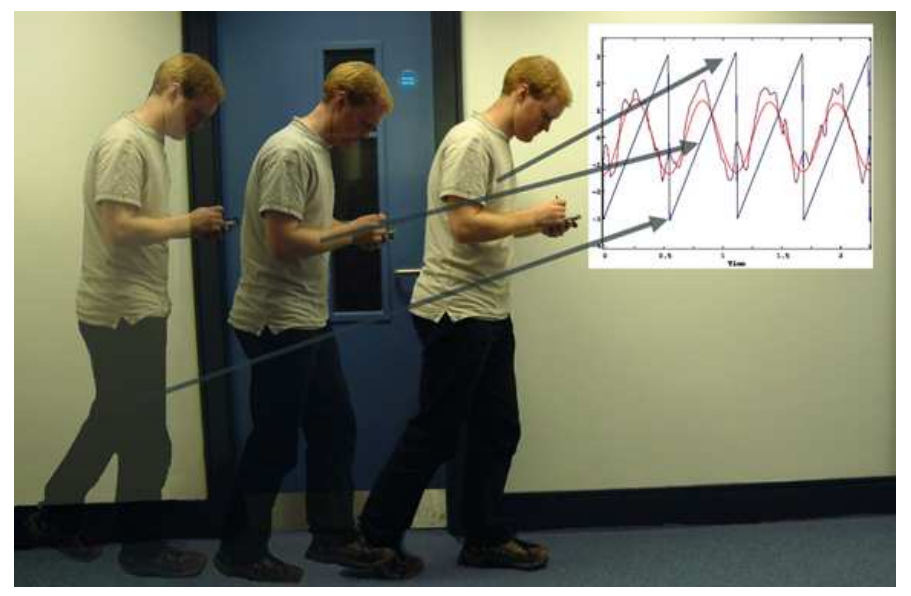

Figure 2. A user walking with the device and corresponding acceleration trace. The unfiltered vertical acceleration signal (rough sinusoid), the filtered signal (smooth sinusoid) and the phase estimate (in radians) for the signal (saw-tooth). 


\section{RESULTS}

\section{Time to Tap}

The mean time to tap was lower in the sitting case than the walking case as would be expected. The mean time to tap a target in the walking condition was $0.79 \mathrm{~s}(\mathrm{std}$. dev. $=0.18$ ) compared to $0.70 \mathrm{~s}$ (std. dev. $=0.22$ ) in the seated case. This can be further broken down into tapping the centre target and outer targets. The mean time to tap the centre target was $0.75 \mathrm{~s}$ (std. dev. $=0.23$ ) when walking and $0.65 \mathrm{~s}$ (std. dev. $=0.19$ ) while sitting. This compared to $0.82 \mathrm{~s}$ (std. dev. $=$ 0.22 ) while walking and $0.75 \mathrm{~s}$ (std. dev. $=0.20$ ) while sitting to tap the outer targets. This difference between centre and outer targets is indicative of users predicting the appearance of the centre target since it consistently appeared every second target.

\section{Tap accuracy}

A graph of tapping accuracy is shown in Figure 3. The graph demonstrates that as expected, users were more accurate tapping in the seated condition with $78 \%$ of taps being within 5 pixels in the seated case compared to $56.5 \%$ in the walking case. Participants remained more accurate in the seated case and reached $98 \%$ of taps within 15 pixels in the seated condition compared to compared to 25 pixels in the walking condition. Separating these into $x$ and $y$ pixel error showed little difference between accuracy in vertical or horizontal error.

Above the range of 30 pixels, structure can be seen in the errors where tap position corresponds to the position of the previous target. This indicates a tap when the user did not mean to tap. This is most likely the result of a user accidently double tapping in position of the previous target. These taps were viewed as outliers and discounted from the final analysis.

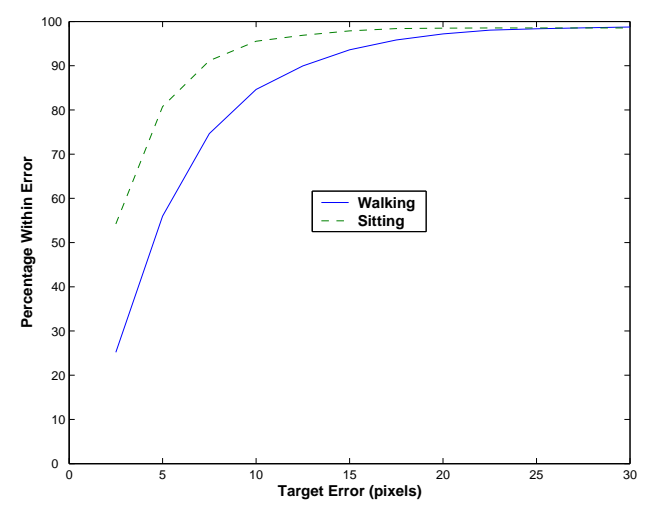

Figure 3. Percentage of taps with the given pixel radius for sitting and walking users.

Observation in the walking condition showed that when tapping, all participants immediately adopted the strategy of grounding the side of their hand holding the stylus on the hand holding the device to reduce independent movement of the hands and thereby improve accuracy. Targeting therefore involve pivoting the hand about the grounded position.

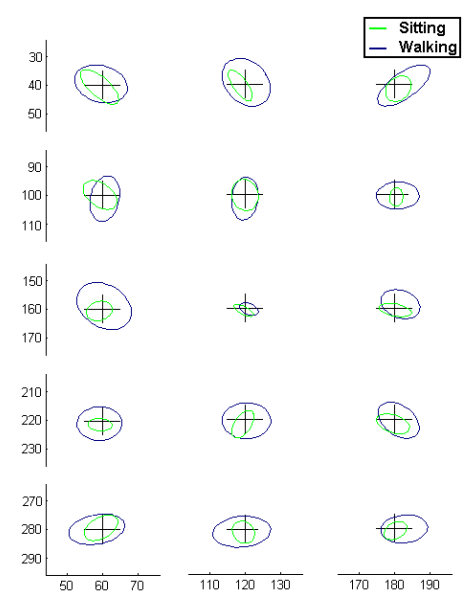

Figure 4. Ellipses show 2 standard deviations of a Gaussian fit to the spread of mean tap positions (from 4 points per participant) from all 20 participants, for each target. In each case the smaller ellipse shows the results for the seated condition and the larger ellipse shows the results for the walking condition. The crosses represent the target positions.

Figure 4 shows the variability and covariance of the $x$ and $y$ target errors for all users for each of the 15 targets. In almost all cases, the variability in tapping is greater in the seated condition than in the walking condition. Due to the controlled conditions of this study, the movements to the outer targets were always from centre target. The variability in tap position for the centre targets is less than that of the outer targets. This is due to the fact that the stylus over the centre target position was the default position for most users. Covariance of the $x$ and $y$ tap positions can be seen to be along the direction of movement for most of the targets. This is particularly true for the corner targets.

\section{Tap Phase}

The method for obtaining the phase of step that the tap occurred is described above. Figure 5 splits one step into 10 equal sections and plots the median of the number of taps in each section for each participant. The reset phase position corresponds to the lowest point of the vertical accelerometer trace. Bins 1 to 5 correspond to the arm as it moves upwards to its peak, and bins 6 to 10 correspond to the arm moving downwards.

A bias is clearly shown towards tapping in the second half of the oscillation. This bias is not present when analysing the phase at which the targets are displayed and must therefore have been introduced by the user. The phases when most taps occur correspond to when the device is moving downwards with the arm. As soon as the device begins to move upwards in the hand again towards the stylus, the number of taps on the screen decreases. When questioned after the experiment, none of the participants was aware that a bias existed.

Figure 6 shows the median of the mean magnitude tap error 


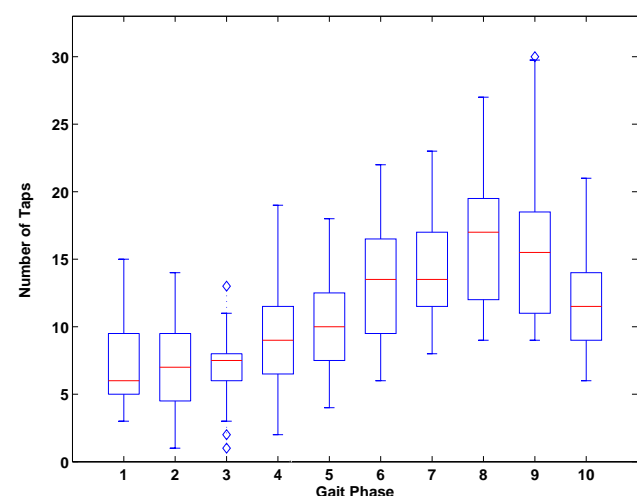

Figure 5. Box plot visualising the distribution of tapping times. Median phase in which the user taps (split into 10 sections)with the reset position for the phase corresponds to the lowest point of the arm which occurs just after a step.

for each participant, for each of the step phase bins above. This figure shows that users were more accurate when tapping in the second half of the phase - the time when most taps occurred. The mean error is 7.1 pixels in the first section (just when the arm starts to rise again), compared to a mean of 5.6 pixels in the fourth section when the hand is moving downwards. When combined with the results shown in Figure 5 above, these data suggest that users were able to unconsciously alter their behaviour in the task in order to improve their accuracy by tapping at a time in their step when it was easier to tap more accurately.

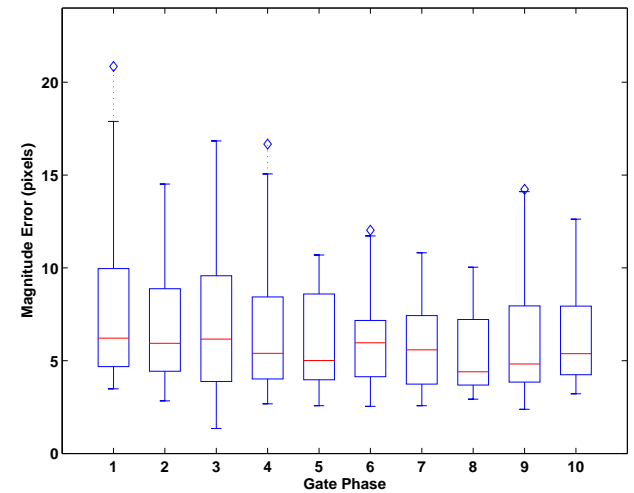

Figure 6. Boxplot showing median target tap error in pixels for each phase of the motion (split into $10 \mathrm{seg}$ ments) with the reset position for the phase at the lowest point of the arm, just after a step. Note the increasing skew to larger errors in less-favoured tapping regions.

\section{CONCLUSIONS AND FUTURE WORK}

This work has demonstrated that by making fine-grained observations from sensors on a mobile device, that we can learn increased detail about the timing and error rates for users. Specific experimental observations of this instrumented usability approach are:

- Users' tapping time is significantly correlated with gait phase angle. User were approximately 3 times more likely to tap at the peak tap phase than the lowest tap phase.

- Users' tapping accuracy is significantly higher (lower mean error and lower variability) at these preferred phase angles. There is further structure in the left step-right step tap density, error biases and variability, but even when averaged over all steps, the results are significant.

- The distribution of tapping errors varies both with phase of step, and between walking and sitting. It also varies across different screen positions. Tapping accuracy was, unsurprisingly, typically greater when sitting still, rather than walking.

This work has relevance for tasks such as text entry in mobile settings. While this work was tap-based, similar features might be found in button-pressing, graffiti gestures or tiltbased interaction. The work also opens new directions in both design and usability areas for future work:

- Does designing an interface such that users tend to tap in preferred phase ranges lead to quantitatively better performance and qualitatively more pleasant user experience? Might it be better to delay user prompts until a particular phase region, in order to sustain rhythmic interaction? This suggests experiments deliberately timing the presentation of prompts, or by using rhythmic vibrotactile or audio feedback in such a way that the user is pushed towards tapping in the specific phase regions.

- The effects of bias and $x-y$ correlation in tapping errors can be systematically compensated for in real time, improving the tapping accuracy. This information can also be used to automatically adapt screen layout to walking speed, simplifying and spreading out the targets as the speed increases.

- We have the opportunity to couple the more objective methods of measuring walking speed used in this paper with the existing literature relating usability to the subjective use of Percentage Preferred Walking Speeds in, e.g. [5].

\section{ACKNOWLEDGEMENTS}

AC \& RM-S are grateful for the support of SFI grant 00/PI.1/C067. RMS, SB, JK \& BM are grateful for support of EPSRC grant GR/R98105/01, and RM-S \& SB BRG project Continuous Gestural Interaction with Mobile devices.

\section{REFERENCES}

1. Brewster, S. A. Overcoming the lack of screen space on mobile computers. Personal and Ubiquitous Computing 6, 3 (2002), 188-205.

2. Lantz, V., and Murray-Smith, R. Rhythmic interaction with a mobile device. NordiCHI '04, Tampere, Finland. ACM (2004), 97-100.

3. Petrie, H., Furner, S., and Strothotte, T. Design lifecycles and wearable computers for users with disabilities. First workshop on human-computer interaction with mobile devices, (Glasgow, UK). Glasgow University (1998).

4. Pikovsky, A., Rosenblum, M., and Kurths, J. Synchronization: A universal concept in nonlinear sciences. Cambridge University Press, 2001.

5. Pirhonen, A., Brewster, S. A., and Holguin, C. Gestural and audio metaphors as a means of control for mobile devices. Proceedings of ACM CHI 2002 (Minneapolis, MN). ACM Press Addison Wesley (2002), 291-298. 